\title{
Small interfering RNA against ERK1/2 attenuates cigarette smoke-induced pulmonary vascular remodeling
}

\author{
MUQING YU, XIANSHENG LIU, HONGXU WU, WANG NI, SHIXIN CHEN and YONGJIAN XU \\ Department of Respiratory and Critical Care Medicine, Tongji Hospital of Tongji Medical College, \\ Huazhong University of Science and Technology, Wuhan, Hubei 430030, P.R. China
}

Received October 19, 2015; Accepted February 27, 2017

DOI: $10.3892 /$ etm.2017.5160

\begin{abstract}
Cigarette smoke may contribute to pulmonary vascular remodeling (PVR), a result of the proliferation of pulmonary artery smooth muscle cells (PASMCs), before pulmonary hypertension in chronic obstructive pulmonary disease (COPD). Activated extracellular signal-regulated kinases 1 and 2 (ERK1/2) are considered to be involved the process of PVR. This study investigated the potential role of ERK1/2 in the proliferation of rat PASMCs (rPASMCs) and cigarette smoke-induced PVR in rats. A small interfering RNA (siRNA) against ERK1/2 (ERK1/2-siRNA) was synthesized, and it significantly reduced the expression of ERK1/2 and cyclin E1, significantly increased the proportion of cells arrested at G0/G1 phase and significantly suppressed the proliferation of rPASMCs treated with cigarette smoke extract compared with controls (all $\mathrm{P}<0.05$ ). In rats, ERK1/2-siRNA, which was administered intranasally, also inhibited the activation of ERK1/2 and the upregulation of cyclin E1, both of which were induced after the rats were exposed to cigarette smoke for 3 months. ERK1/2-siRNA also significantly reduced PVR (observed by vessel wall thickness and the proportion of fully muscularized vessels) in cigarette smoke-exposed rats compared with a negative control siRNA $(\mathrm{P}<0.05)$. Collectively, these data indicated that ERK1/2-siRNA could attenuate PVR in cigarette smoke-exposed rats, and it may have therapeutic value in the treatment of COPD.
\end{abstract}

Correspondence to: Professor Yongjian $\mathrm{Xu}$, Department of Respiratory and Critical Care Medicine, Tongji Hospital of Tongji Medical College, Huazhong University of Science and Technology, 1095 Jiefang Road, Wuhan, Hubei 430030, P.R. China E-mail: yjxu@tjh.tjmu.edu.cn

Abbreviations: PVR, pulmonary vascular remodeling; COPD, chronic obstructive pulmonary disease; rPASMCs, rat pulmonary artery smooth muscle cells; CSE, cigarette smoke extract; ERK, extracellular signal-regulated kinase

Key words: chronic obstructive pulmonary disease, cigarette smoke, cyclin E1, extracellular signal-related kinase 1/2, pulmonary vascular remodeling

\section{Introduction}

Chronic cigarette smoke is the most important risk factor for the development of chronic obstructive pulmonary disease (COPD) (1). Previous results have indicated that smoking may induce pulmonary vascular remodeling (PVR), which leads to increased pressure in the pulmonary artery (2-5), suggesting that smoking might directly result in PVR at the initial stage of COPD $(5,6)$. Increased proliferation of pulmonary arterial smooth muscle cells (PASMCs) directly caused by cigarette smoke has been reported by previous studies $(2,7)$, and is considered to be one of the pathological foundations of PVR and irreversible pulmonary hypertension. However, the molecular mechanisms underlying this process remain unclear.

It has previously been reported that cigarette smoke can exert biological effects in an extracellular signal-regulated kinase 1 and 2 (ERK1/2)-dependent manner (8). ERK1/2 are members of the large family of mitogen-activated protein kinases. Activation of ERK1/2 (p-ERK1/2) has been implicated as a regulator in numerous fundamental cellular activities $(9,10)$. Studies by the current authors and other researchers have addressed the role of the ERK1/2 signaling pathway in the proliferation of airway smooth muscle cells and multiple arterial smooth muscle cells (11-14).

Cell cycle progression is regulated precisely at various biological checkpoints by cyclins, cyclin-dependent kinases (CDK) and CDK inhibitors (15). Cyclin E1, the major E-type cyclin, is expressed during late G1 phase until the end of S-phase (16). The activity of cyclin E1 regulates cells passing the restriction point ' $R$ ', which marks a 'point of no return' in cell cycle transition from a resting state into the division cycle or from G1 phase into S phase (16). Previous studies have reported that the expression of cyclin E1 could be upregulated by activated ERK1/2 (17-21). A previous study by the current authors indicated that cyclin E1 might participate in ERK-related PVR (22). However, further investigation is required to determine whether the ERK1/2-cyclin E1 signaling pathway is involved in PVR induced by cigarette smoke.

To investigate the role of the ERK1/2-cyclin E1 signaling pathway in PVR induced by cigarette smoke, the expression of ERK1/2 and cyclin E1 in cultured rat PASMCs (rPASMCs) and rat pulmonary arteries, as well as the structural changes of pulmonary vessels, were evaluated in the presence of ERK1/2-small interfering RNA (siRNA). The results indicated an attenuation 
of PVR by ERK1/2-siRNA in cigarette smoke-exposed rats, suggesting that ERK1/2-siRNA may have therapeutic value in the treatment of COPD.

\section{Materials and methods}

Reagents. All siRNAs were purchased from Guangzhou RiboBio Co., Ltd. (Guangzhou, China). The siRNA sequences were as follows: ERK1, 5'-GACCGGAUGUUAACCUUUA-3'; ERK2, 5'-CCAGGAUACAGAUCUUAAA-3'; and negative control, 5-UUCUCCGAACGUGUCACGU-3'. All siRNAs (final concentration $30 \mathrm{nmol} / \mathrm{l}$ ) were transfected into rPASMCs with Lipofectamine 2000 (Invitrogen; Thermo Fisher Scientific, Inc., Waltham, MA, USA). For in vivo studies, each rat was anesthetized with diethyl ether and then administered intranasally with ERK1/2-siRNA $(15 \mathrm{nmol})$ or an equivalent dose of the negative control siRNA twice a week. The anesthetization procedure was performed as follows: The rat was placed into a glass jar with 1-1.5 ml diethyl ether. After $60-90 \mathrm{sec}$, the inhalation of the diethyl ether led to the anesthetization of the rat. Anesthetization was confirmed by swaying the glass jar.

The antibody against p-ERK (9101) was purchased from Cell Signaling Technology Inc. (Danvers, MA, USA), the antibody against cyclin E1 (ab7959) was purchased from Abcam (Cambridge, UK) and the antibody against ERK1/2 (SC-153) was purchased from Santa Cruz Biotechnology, Inc. (Dallas, TX, USA).

Total RNA was isolated using TRIzol reagent (Invitrogen; Thermo Fisher Scientific, Inc.) according to the manufacturer's instructions. First-strand cDNA synthesis was performed with PrimeScript $\mathrm{RT}^{\mathrm{TM}}$ reverse transcriptase (Takara Biotechnology Co., Ltd., Dalian, China). The primers used for real-time detection were as follows: ERK1 (243 bp), forward, 5'-AGAATGTCATAGGCATCCGAGA-3' and reverse, 5'-CGCAGGTGGTGTTGATAAGC-3'; ERK2 (201 bp), forward: 5'-ACCTCAAGCCTTCCAACCTC-3' and reverse, 5'-AGCCCACAGACCAAATATCAAT-3'; cyclin E1 (176 bp), forward, 5'-GGAAAATCAGACCGCCCAG-3' and reverse, 5'-CATCAGCCAGTCCAGAAGAAC-3'; $\beta$-actin (110 bp), forward, 5'-CGTTGACATCCGTAAAGACCTC-3' and reverse, 5'-TAGGAGCCAGGGCAGTAATCT-3'.

Animals and cigarette smoke exposure. A total of 24 adult male Wistar rats (age, 8-12 weeks; weight, 200-250 g at the start of this study), obtained from the Animal Application Center at Tongji Hospital (Wuhan, China), were bred in a barrier system at room temperature $\left(18-23^{\circ} \mathrm{C}\right)$ and $40-70 \%$ humidity. The rats were fed with food $(5 \mathrm{~g} / 100 \mathrm{~g}$ weight) and water $(10 \mathrm{ml} / 100 \mathrm{~g}$ weight $)$ every day in a 12-h light/12-h dark cycle. Rats were randomly divided into four groups: Control, smoking, smoking + negative control (NC)-siRNA and smoking + ERK1/2-siRNA. Rats were exposed to normal air or the smoke of 20 Marlboro cigarettes (Philip Morris USA; Altria Group, Inc., Richmond, VA, USA) per day for three months. The cigarette smoke exposure was carried out with a PAB-S200 Animal Passive Smoking Exposure System (BioLabs Technology Co., Ltd., Beijing, China).

The study was conducted according to the principles of the Declaration of Helsinki and approved by the Institutional
Review Board of Tongji Hospital of Tongji Medical College, Huazhong University of Science and Technology in accordance with its guidelines for the protection of animal subjects. The Institutional Review Board approved the animal experiments for this research.

Cell culture. Primary rPASMCs were prepared from explants of endothelium and adventitia-stripped intra-pulmonary arteries of 6 additional Wistar rats (Male, 6-8 weeks, same source and housing conditions as above) with an average body weight of $220 \mathrm{~g}$ (200-250 g). The rats were sacrificed by the intraperitoneal injection of sodium pentobarbital $(150 \mathrm{mg} / \mathrm{kg})$, and the explants and primary rPASMCs were obtained as previously described (23). Cells were cultured in Dulbecco's modified Eagle's medium (Gibco; Thermo Fisher Scientific, Inc.) with $10 \%$ heat-inactivated fetal bovine serum (Gibco; Thermo Fisher Scientific, Inc.) and identified by phase-contrast microscopy and immunochemical staining. Cells from passage 3-8 were used for all experiments.

Immunochemical staining. Cells were fixed with $4 \%$ paraformaldehyde for $20 \mathrm{~min}$ at room temperature. Nonspecific binding sites were blocked in $10 \%$ bovine serum in phosphate-buffered saline (PBS) at room temperature for $1 \mathrm{~h}$ and cells were immunostained with anti- $\alpha$-smooth muscle actin (SMA) primary antibody (1:200 dilution; sc-32251; Santa Cruz Biotechnology, Inc.) for $1 \mathrm{~h}$ at room temperature, followed by reaction with biotinylated secondary antibody (biotin-conjugated affinipure goat anti-mouse IgG; SA00004-1; Proteintech Group, Inc., Chicago, IL, USA) for 30 min (1:200 dilution) at room temperature. Subsequently, cells were incubated with HRP-conjugated streptavidin (cat. 434323, Thermo Fisher) for $30 \mathrm{~min}$ at room temperature (1:200 dilution) and the appropriate substrate solution (3,3'-diaminobenzidine substrate solution; 34002; Pierce; Thermo Fisher Scientific, Inc.) was added and treated for 5-15 min. Images were captured under a fluorescence microscope (Nikon Corporation; Tokyo, Japan).

Western blotting. Cells were lysed by lysing buffer $(50 \mathrm{mM}$ Tris-HCl, pH 7.5, $300 \mathrm{mM} \mathrm{NaCl}, 1 \%$ Triton-X, 5 mM EDTA and $10 \%$ glycerol) and the whole proteins were quantified using a commercial BCA assay kit (23225; Pierce; Thermo Fisher Scientific, Inc.) according to the manufacturer's instructions. Cell lysates were loaded (30 $\mu$ g per lane) and electrophoresed in 12\% SDS-PAGE and transferred onto a polyvinylidene difluoride membrane. At room temperature, the membrane was incubated in a 5\% non-fat milk- tris-buffered saline with Tween-20 solution for at least 30 min to block non-specific binding. The membrane was incubated with the primary antibody (1:1,000 dilution) for $2 \mathrm{~h}$ at room temperature. The following primary antibodies were used to detect various target proteins: The antibody against p-ERK (9101; Cell Signaling Technology, Inc.), against cyclin E1 (ab7959; Abcam), against ERK1/2 (SC-153; Santa Cruz Biotechnology, Inc.) and the antibody against $\beta$-actin (60008-1-Ig; Proteintech Group, Inc.). Then the membrane was incubated with the appropriate horseradish peroxidase-conjugated secondary antibody (1:5,000 dilution; goat anti-rabbit IgG; SA00001-2; Proteintech Group, Inc.) for $45 \mathrm{~min}$ at room temperature. 
The membrane was subsequently incubated with enhanced chemiluminescence substrate (32106; Pierce; Thermo Fisher Scientific, Inc.) for $5 \mathrm{~min}$, and the result was detected by a luminescent image analyzer (LAS4000; Fujifilm; Tokyo, Japan).

Reverse transcription-quantitative polymerase chain reaction (RT-qPCR) analysis. Whole RNA was extracted from the cells using TRIzol reagent (Invitrogen; Thermo Fisher Scientific, INc.) and reversed transcribed into cDNA using reverse transcriptase (M-MLV; M170A; Promega Corporation; Madison, WI, USA) and Oligo dT for $1 \mathrm{~h}$ at $42^{\circ} \mathrm{C}$. qPCR was performed using the SYBR Green method and the commercial kit ( $\mathrm{iQ}^{\mathrm{TM}}$ SYBR $^{\circledR}$-Green Supermix; 1708880; Bio-Rad Laboratories, Inc., Hercules, CA, USA) on ABI-7500 (Life Technology). Samples were prepared according to the instructions of the manufacturer of the $\mathrm{iQ}^{\mathrm{TM}} \mathrm{SYBR}^{\circledR}$ Green Supermix and the qPCR programmed was set as follows: Denaturation at $95^{\circ} \mathrm{C}$ for $3 \mathrm{~min}$, followed by 40 cycles of amplification (denaturation at $95^{\circ} \mathrm{C}$ for $15 \mathrm{sec}$, annealing and extension at $60^{\circ} \mathrm{C}$ for $30 \mathrm{sec}$; the plate was read at the end of each extension phase), melt curve analysis was conducted from 55 to $95^{\circ} \mathrm{C}$ with continuous plate reading. The result was automatically calculated by the $2^{-\Delta \Delta \mathrm{Cq}}$ method (24) on ABI-7500 following the completion of the experiment.

Preparation of cigarette smoke extract (CSE). Smoke from two commercial Marlboro cigarettes was bubbled through $50 \mathrm{ml}$ phosphate-buffered saline (PBS). After the solution was adjusted to $\mathrm{pH} 7.4$ and filtered through a $0.22-\mu \mathrm{m}$ sterile filter, it was then defined as $100 \%$ CSE. We previously determined that $2 \%$ CSE had the strongest effect on promoting the proliferation of rPASMCs (25), so this concentration of CSE was used for the entire in vitro study. Cells were starved for $24 \mathrm{~h}$ in serum-free medium at $37^{\circ} \mathrm{C}$ before they were treated with CSE or an equal amount of PBS. At $24 \mathrm{~h}$, cells were harvested for subsequent experiments.

Cell proliferation assays. Cell proliferation was assessed by cell counting and 5-bromo-2-deoxyuridine (BrdU; Roche Diagnostics GmbH, Mannheim, Germany) incorporation assay. The rPASMCs were seeded into 24-well plates at a density of 3,000 cells/well. After the aforementioned treatment with CSE or PBS, cells were counted using a hemocytometer (wi81386; Dongxiyi Company, China) by trypan blue exclusion.

The BrdU incorporation assay was performed to detect active DNA synthesis. Briefly, cells were plated on cover slips and treated with CSE or PBS. They were then incubated with $50 \mathrm{mmol} \mathrm{BrdU}$ for $4 \mathrm{~h}$ at $37^{\circ} \mathrm{C}$ and fixed with cold acetone. The cells were then permeabilized and counted under a fluorescence microscope. The results of the BrdU incorporation assay were presented as the percentage of BrdU-positive cells. The detailed procedure has been described previously (25).

Cell cycle analysis by flow cytometry. After treatment with CSE or PBS, $1 \times 10^{6}$ cells $/ \mathrm{ml}$ were immediately digested by EDTA-free trypsin and fixed overnight at $4^{\circ} \mathrm{C}$ in $70 \%$ ethanol. Then, cells were suspended in PBS containing propidium iodide $(0.5 \mathrm{mg} / \mathrm{ml}$; Sigma-Aldrich; Merck KGaA, Darmstadt, Germany) and DNase-free RNase $(0.1 \mathrm{mg} / \mathrm{ml})$. Flow cytometry was performed using a FACSort system with CellQuest software (version 5.1; BD Biosciences, Franklin Lakes, NJ, USA). The percentages of cells in different phases of the cell cycle were determined using ModFit software (version 3.2; Verity Software House, Inc., Topsham, ME, USA).

Tissue preparation. Rats were provided with fresh air for $24 \mathrm{~h}$ after the last exposure to cigarette smoke and then anesthetized with pentobarbital $(50 \mathrm{mg} / \mathrm{kg}$, intraperitoneal). The rats were sacrificed by exsanguination, then the lungs of all rats were collected. The intrapulmonary arteries were isolated from left lungs for western blot and reverse transcription-quantitative polymerase chain reaction (RT-qPCR) analysis. The right lungs were fixed in $4 \%$ paraformaldehyde overnight, embedded in paraffin and cut into $5-\mu \mathrm{m}$ sections for morphological examination.

Morphometric analysis of pulmonary vessels. Sections of the right lungs were stained with hematoxylin and eosin to observe morphological changes in the small pulmonary arteries. For each rat, five arteries with an external diameter between 50 and $150 \mu \mathrm{m}$ were randomly selected and evaluated using a HPIAS-1000 medical image-analysis system (Wuhan Champion Image Engineering Co., Wuhan, China). The detailed selection procedure was as follows: Two technicians each selected five arteries from five different visual fields with an optical microscope. Ten arteries were selected in total, then five arteries were randomly selected from this pool. The wall area and total area of the arteries were measured. Wall thickness $($ WT $)=($ Wall area/total area) $x 100 \%$ (26). All morphometric measurements were performed by two technicians operating in a blind manner. The difference in measurements between the two observers was $<5 \%$.

Assessment of PVR. Further evaluation of PVR was performed by immunostaining with an anti- $\alpha$-SMA antibody (sc-32251; 1:200 dilution; Santa Cruz Biotechnology, Inc.). All small intrapulmonary arteries $(\leq 200 \mu \mathrm{m}$ in external diameter) adjacent to the alveolar ducts were analyzed. The fully muscularized vessels (actin staining $>75 \%$ of the circumference) were counted and the result was presented as the percentage of the total small intrapulmonary arteries.

Immunostaining of ERK1/2 and cyclin E1. As previously described (27), sections were deparaffinized, rehydrated, soaked in an antigen retrieval solution with citrate buffer, and incubated overnight at $4^{\circ} \mathrm{C}$ with a rabbit polyclonal antibody against p-ERK (9101; 1:200 dilution; Cell Signaling Technology) or cyclin E1 (ab7959; 1:200 dilution; Abcam). Biotinylated secondary antibody (SA00004-1; 1:200 dilution; Proteintech Group, Inc.) was then added and followed by incubation with a streptavidin-peroxidase conjugate for $30 \mathrm{~min}$ at room temperature. Images were captured under a fluorescence microscope (Nikon Corporation).

Statistical analysis. Data are presented as the mean \pm standard deviation. Statistical software (PASW Statistics 18.0; IBM SPSS, Armonk NY, USA) was used for analysis and the Student's t-test was performed. $\mathrm{P}<0.05$ (two-sided) was considered to indicate a statistically significant result. 
A

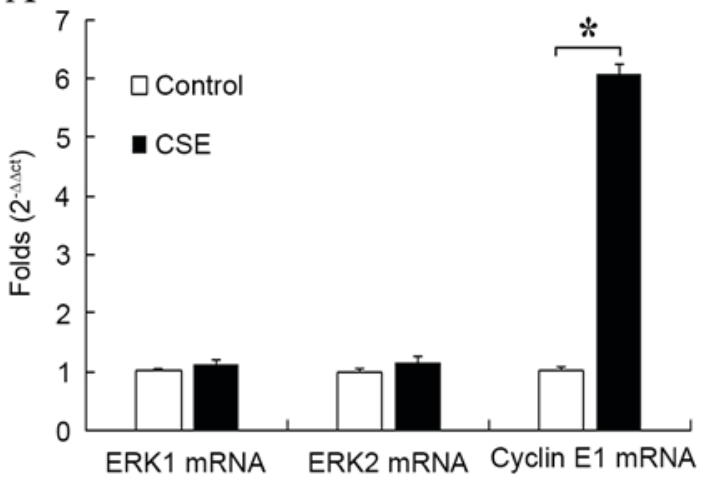

$\mathrm{C}$

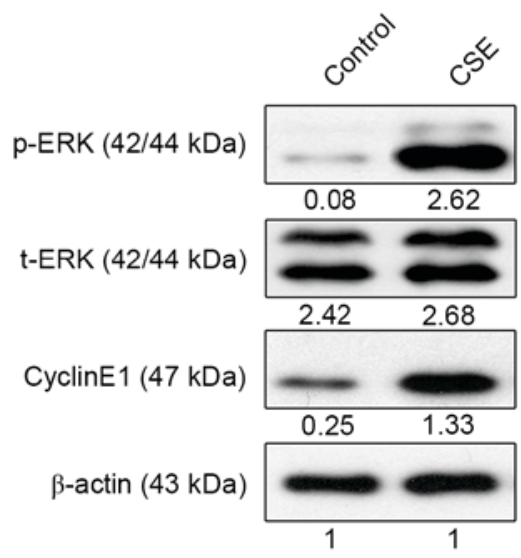

B

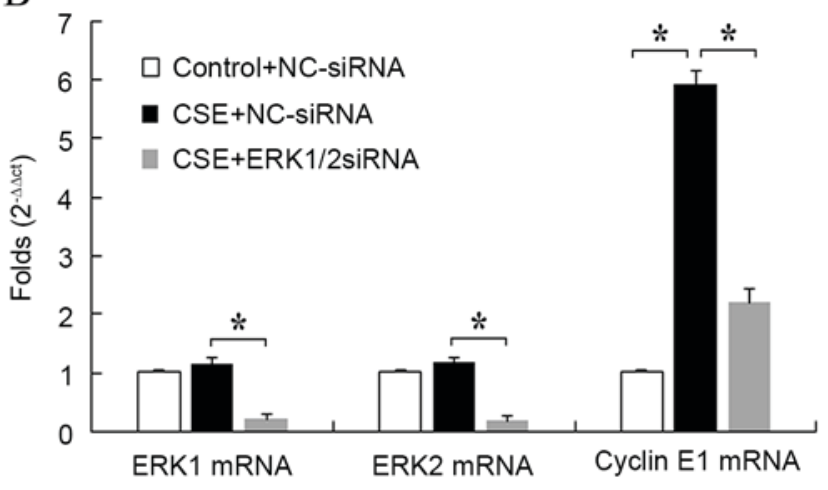

$\mathrm{D}$

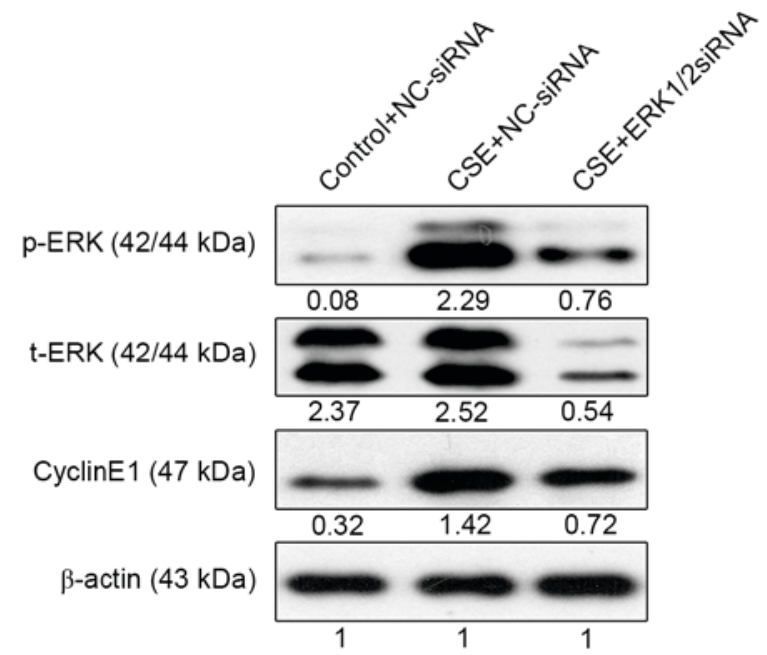

Figure 1. Effect of ERK1/2-siRNA on the expression of cyclin E1 induced by CSE. (A) Cells were treated with CSE or the control medium for $24 \mathrm{~h}$, then the mRNA levels of ERK1/2 and cyclin E1 were determined by RT-qPCR. (B) Cells were transfected with ERK1/2-siRNA or NC-siRNA at a final concentration of $30 \mathrm{nmol} / 1$ for $24 \mathrm{~h}$, then the cells were treated with CSE or the control medium for another $24 \mathrm{~h}$. The mRNA levels of ERK1/2 and cyclin E1 were determined by RT-qPCR. (C) Cells were treated with CSE or the control medium for $24 \mathrm{~h}$, then the protein levels of ERK1/2 and cyclin E1 were determined by western blot analysis. (D) Cells were treated as in (B), then the protein levels of ERK1/2 and cyclin E1 were determined by RT-qPCR. "P<0.05. ERK, extracellular signal-regulated kinase; siRNA, small interfering RNA; CSE, cigarette smoke extract; RT-qPCR, reverse transcription-quantitative polymerase chain reaction; $\mathrm{NC}$, negative control.

\section{Results}

ERK1/2-siRNA suppresses the expression of ERK1/2 and cyclin El in CSE-treated rPASMCs. Rat PASMCs were treated with $2 \%$ CSE for $24 \mathrm{~h}$, then the mRNA expression of ERK1/2 and cyclin E1 were evaluated by RT-qPCR. Compared with the control, CSE significantly increased the mRNA expression of cyclin E1 (P<0.05; Fig. 1A). However, it had no influence on the mRNA expression of ERK1/2.

Next, the effect of ERK1/2-siRNA on the expression of ERK1/2 and cyclin E1 during CSE treatment was detected. As shown in Fig. 1B, mRNA expression of cyclin E1 was significantly increased after CSE treatment compared with the control $(\mathrm{P}<0.05)$. However, this increase was significantly attenuated by treatment with ERK1/2-siRNA $(\mathrm{P}<0.05)$. This result suggested that ERK1/2-siRNA could block the effect of CSE on the mRNA expression of cyclin E1. The mRNA expression of ERK1/2 was also evaluated to verify the function of ERK1/2-siRNA. For both ERK1 and ERK2, mRNA expression was significantly reduced in CSE + ERK1/2-siRNA-treated cells compared with CSE + NC-siRNA-treated cells (both $\mathrm{P}<0.05)$.
The effect of CSE on ERK1/2 and cyclin E1 expression was also evaluated at the protein level. Treatment with CSE increased the protein level of cyclin E1 (Fig. 1C), which was consistent with the results at the mRNA level. Also, the expression of p-ERK, but not t-ERK, was markedly upregulated by CSE treatment, which suggested that CSE could stimulate the phosphorylation of ERK1/2 and activate the ERK1/2 signaling pathway (Fig. 1C).

The effect of ERK1/2-siRNA during CSE treatment was also detected at the protein level. Consistent with the results at the mRNA level, cyclin E1 expression was increased by CSE + NC-siRNA treatment and attenuated by CSE + ERK1/2-siRNA treatment (Fig. 1D). ERK1/2-siRNA also markedly reduced the protein levels of both t-ERK and p-ERK. The results of Fig. 1 suggested that CSE could increase the expression of cyclin E1 through activation of the ERK1/2 signaling pathway.

Notably, although CSE-induced expression of cyclin E1 was suppressed by ERK1/2-siRNA at both the mRNA and protein level, its expression was still higher than that in the control group (Fig. 1B and D). This was further confirmed by a dose-response curve experiment (Fig. 2). It was found that the 
A

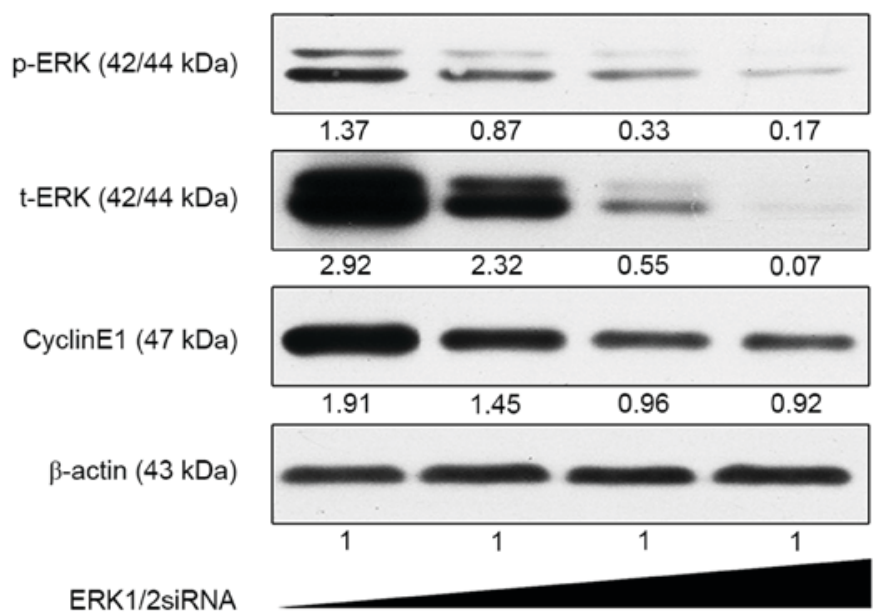

$\mathrm{B}$
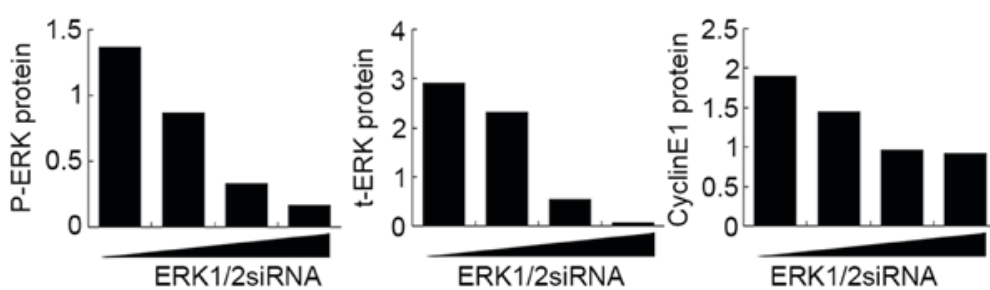

Figure 2. Dose-response curve of ERK1/2-siRNA in rat pulmonary artery smooth muscle cells. Cells were transfected with ERK1/2-siRNA at a final concentration of 0,10,20 and $30 \mathrm{nmol} / 1$ (presented left to right). The protein levels of ERK1/2 and cyclin E1 were determined by western blot analysis. (A) The optical density of each band was detected and normalized against $\beta$-actin. (B) Normalized optical density of each band. ERK, extracellular signal-regulated kinase; siRNA, small interfering RNA.

expression of cyclin E1 no longer decreased as the concentration of ERK1/2-siRNA was increased above $20 \mathrm{nmol} / 1$, whereas the ERK1/2 expression continued to decrease steadily. This result suggested that the ERK1/2 signaling pathway is not the only pathway involved in the upregulation of cyclin E1 by CSE.

ERK1/2-siRNA inhibits progression from G1 phase to $S$ phase and suppresses rPASMC proliferation induced by CSE. The distribution of cells in different cell cycle stages was detected after CSE treatment. A significantly decreased proportion of cells in G0/G1 phase and a significantly increased proportion of cells in $\mathrm{S}$ phase were observed in the CSE-treated group compared with the control $(\mathrm{P}<0.05$; Fig. $3 \mathrm{~A})$. Furthermore, the effect of ERK1/2-siRNA during CSE treatment was evaluated. The results indicated that ERK1/2-siRNA could significantly attenuate the effects of CSE on the cell cycle ( $\mathrm{P}<0.05$; Fig. 3B). Cell proliferation was also evaluated by cell counting (Fig. 3C and D) and BrdU incorporation (Fig. 3E and F). It was found that CSE significantly promoted the proliferation of rPASMCs compared with the control ( $\mathrm{P}<0.05$; Fig. $3 \mathrm{C}$ and $\mathrm{E})$, and this effect was significantly reduced but not abolished by ERK1/2-siRNA $(\mathrm{P}<0.05$; Fig. 3D and F).

ERK1/2-siRNA reduces the expression of ERK1/2 and cyclin $E 1$ in the pulmonary vessels of cigarette smoke-exposed rats. Consistent with the results in vitro, cigarette smoke exposure significantly increased the mRNA expression of cyclin E1 in rat pulmonary arteries compared with the control group $(\mathrm{P}<0.05$; Fig. 4A), whereas the mRNA expression levels of ERK1 and
ERK2 were not influenced. Furthermore, the mRNA level of cyclin E1 induced by the cigarette smoke was significantly reduced after the transfection of ERK1/2-siRNA $(\mathrm{P}<0.05$; Fig. 4A). The mRNA levels of ERK1 and ERK2 were also significantly reduced after transfection with ERK1/2-siRNA (both $\mathrm{P}<0.05$ ), which confirmed the function of ERK1/2-siRNA. When compared with the control group, protein expression cyclin E1 and p-ERK, but not t-ERK, were all markedly increased in the smoking group, and ERK1/2-siRNA inhibited the increased expression induced by cigarette smoke (Fig. 4B). However, the protein expression of cyclin E1 in the ERK1/2-siRNA group was still slightly increased compared with the control group. The immunostaining of p-ERK (Fig. 4C) and cyclin E1 (Fig. 4D) in rat lung histologic sections were consistent with the results at mRNA and protein levels.

ERK1/2-siRNA ameliorates rat PVR induced by cigarette smoke. The vessel wall thickness and the number of fully muscularized vessels are key indicators of PVR (28). Analysis of vessel wall thickness revealed a significant increase in the smoking group compared with the control group $(\mathrm{P}<0.05)$ and a significant decrease in the smoking + ERK1/2-siRNA group compared with the smoking $+\mathrm{NC}$-siRNA group $(\mathrm{P}<0.05$; Fig. 5A). Furthermore, it was indicated that cigarette smoke significantly increased the proportion of fully muscularized vessels in rats compared with the control group, and smoking + ERK1/2-siRNA significantly reduced this proportion compared with the smoking $+\mathrm{NC}$-siRNA group $(\mathrm{P}<0.05$; Fig. 5B). 

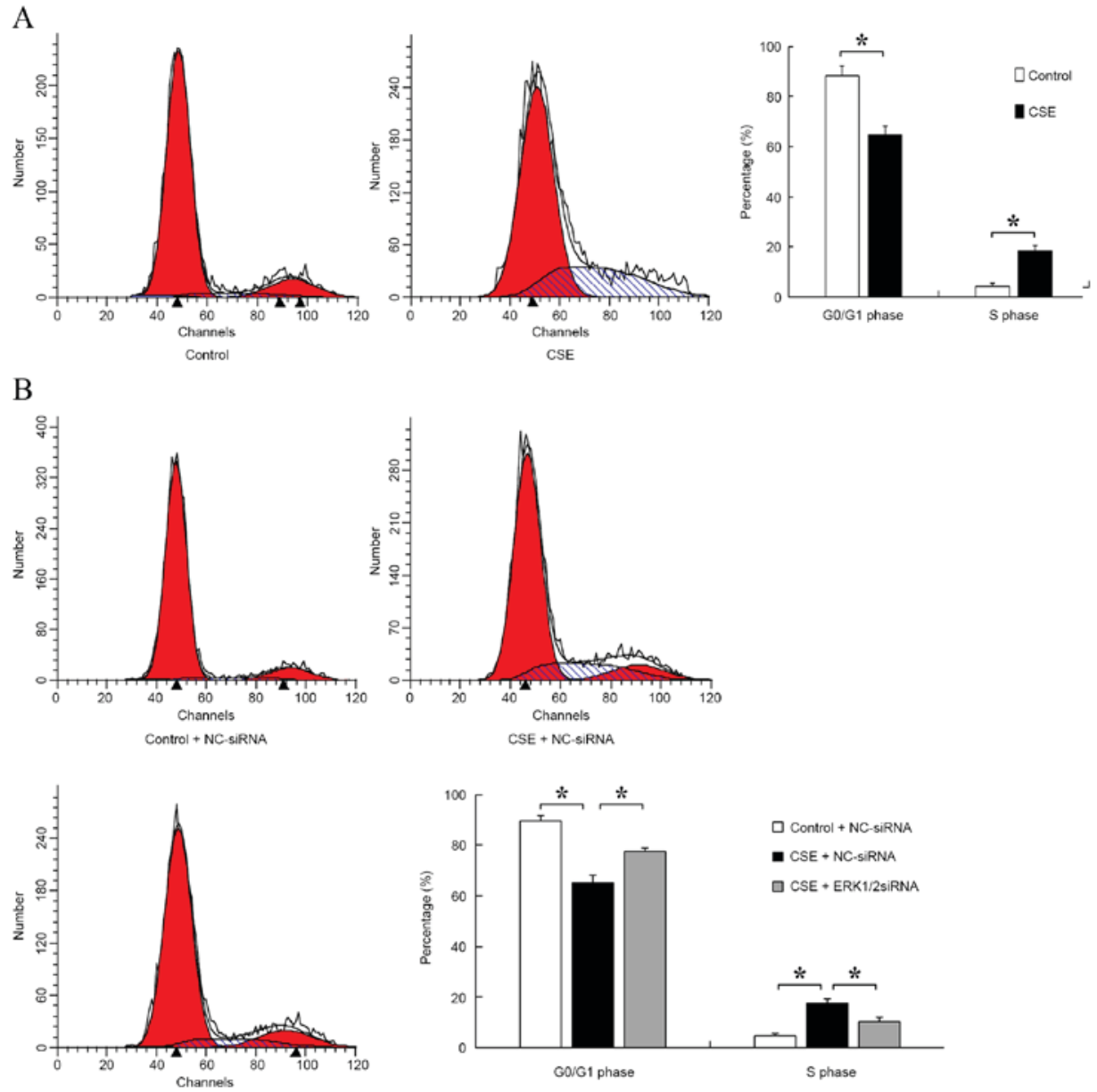

C

CSE + ERK1/2siRNA

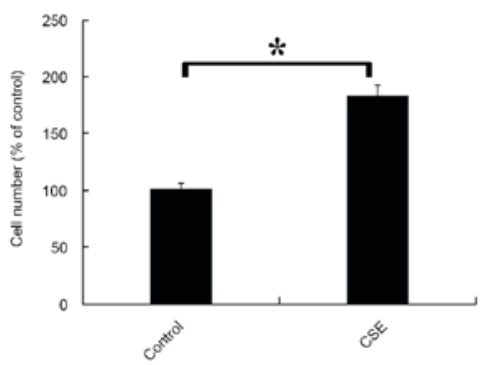

E

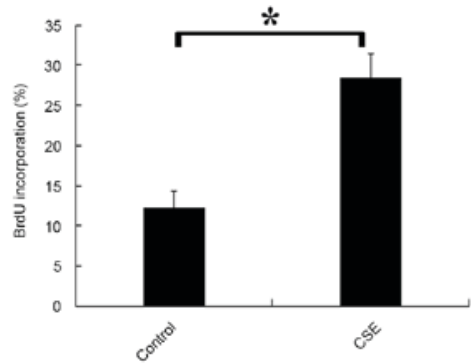

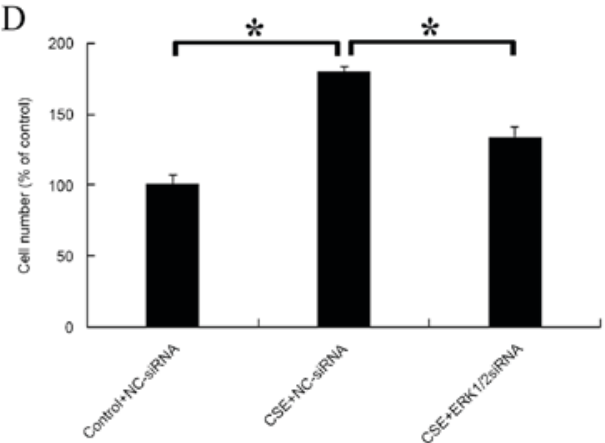

$\mathrm{F}$

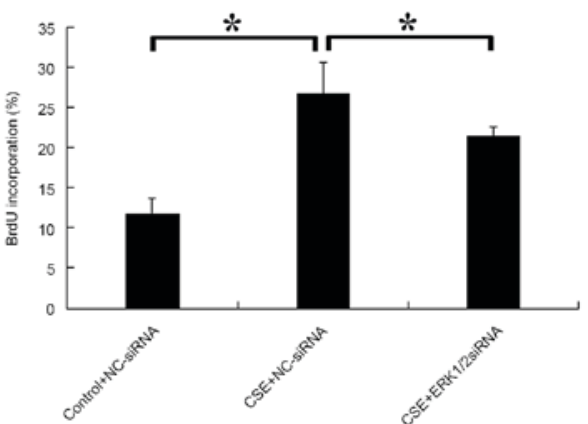

Figure 3. Effect of CSE and ERK1/2-siRNA on rat pulmonary artery smooth muscle cell cycle and cell proliferation. (A) Cells were treated with CSE or the control medium for $24 \mathrm{~h}$, then the distribution of cell cycle stages was detected. The left red, right red and blue areas represent G0/G1, G2/M and S phase, respectively. (B) Cells were transfected with ERK1/2-siRNA or NC-siRNA at a final concentration of $30 \mathrm{nmol} / 1$ for $24 \mathrm{~h}$, then cells were treated with CSE or control medium for a further $24 \mathrm{~h}$. (C) Cells were treated with CSE or the control medium for $24 \mathrm{~h}$, then the cell number of each group was counted. (D) Cells were treated as in (B), then the cell number of each group was counted. (E) Cells were treated with CSE or the control medium for $24 \mathrm{~h}$, then incubated with $50 \mathrm{mmol} \mathrm{BrdU}$ for $4 \mathrm{~h}$ at $37^{\circ} \mathrm{C}$ and fixed with cold acetone. The BrdU-positive cells were counted. (F) Cells were treated as in (B), then incubated with $50 \mathrm{mmol}$ $\mathrm{BrdU}$ for $4 \mathrm{~h}$ at $37^{\circ} \mathrm{C} .{ }^{*} \mathrm{P}<0.05$ vs. control. ERK, extracellular signal-regulated kinase; siRNA, small interfering RNA; CSE, cigarette smoke extract; NC, negative control; BrdU, 5-bromo-2-deoxyuridine. 
A

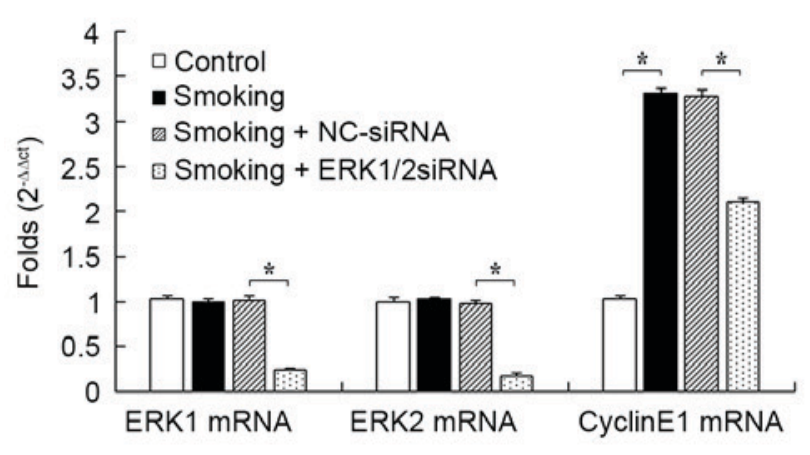

$\mathrm{C}$
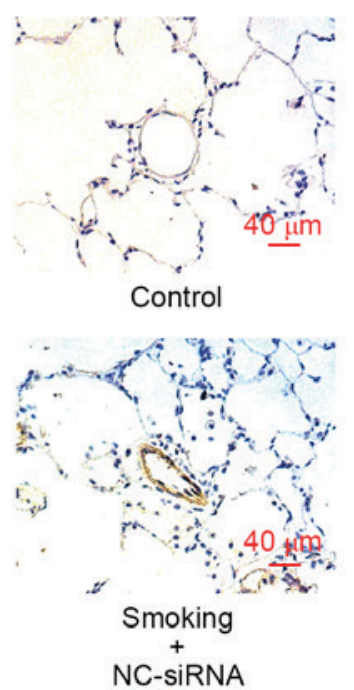
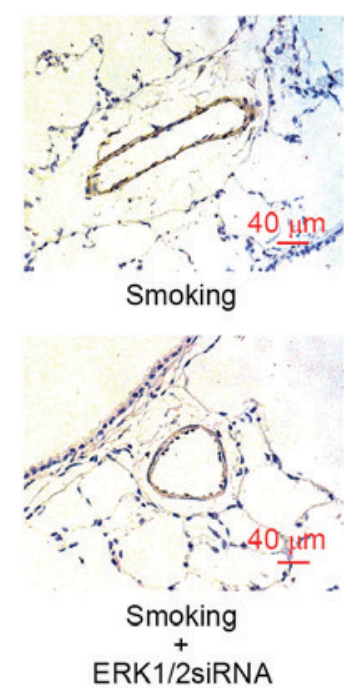

B

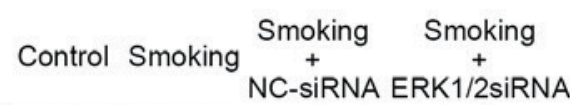

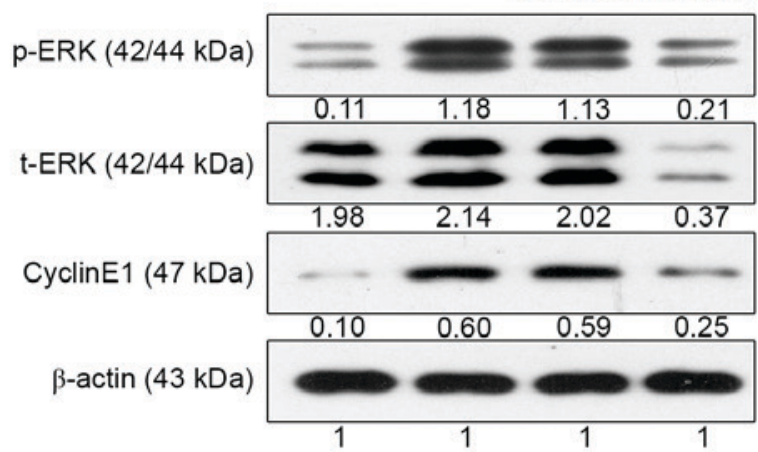

$\mathrm{D}$

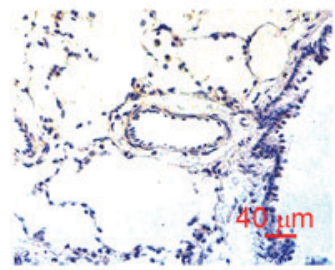

Control

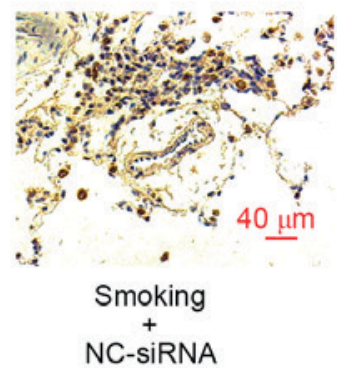

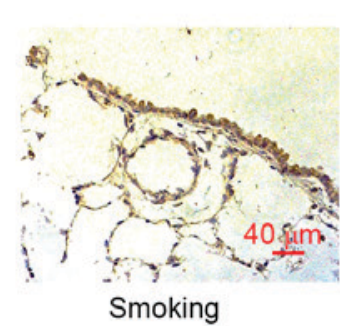

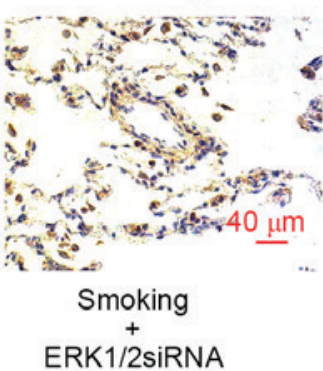

Figure 4. Effect of ERK1/2-siRNA on the expression of ERK1/2 and cyclin E1 in the pulmonary vessels of cigarette smoke-exposed rats. (A) The mRNA levels of ERK1/2 and cyclin E1. (B) Protein levels of ERK1/2 and cyclin E1 were evaluated by western blot analysis. Immunostaining for (C) p-ERK1/2 and (D) cyclin E1 was also performed in each group (C) p-ERK or (D) cyclin E1. Blue, indicated the nucleus; red, indicated the cytoplasm; and dark brown indicated p-ERK or cyclin E1. Magnification, x200. *P<0.05 vs. control. ERK, extracellular signal-regulated kinase; siRNA, small interfering RNA; NC, negative control.

\section{Discussion}

The present study indicated that the knockdown of ERK1/2 expression by siRNA suppressed rPASMC proliferation induced by CSE and moderated PVR in rats exposed to cigarette smoke. It was also observed that ERK1/2-siRNA reduced the G1/S transition induced by CSE via the reduction of cyclin E1 expression. These results suggested that the ERK1/2 signaling pathway is at least partially implicated in the abnormal proliferation of rPASMCs (induced by CSE) and PVR in rats (caused by cigarette smoke exposure), through the regulation of cyclin E1 expression.

In the present study, ERK1/2-siRNA was used to silence the gene expression of ERK1/2 in vitro and in vivo. It was found that $\mathrm{t}$-ERK1/2 was expressed stably in rPASMCs treated with CSE or pulmonary vessels of cigarette smoke-exposed rats at both the mRNA and protein level, compared with control groups. However, p-ERK1/2 was significantly upregulated in those groups, suggesting that ERK1/2 could be activated by cigarette smoke. ERK1/2-siRNA reduced p-ERK1/2 expression in vitro and in vivo at both the mRNA and protein level, by knocking down t-ERK1/2 expression but not the ratio of p-ERK/t-ERK.
Activated ERK1/2 performs multiple cellular functions, including cell proliferation, adhesion and migration $(9,10)$. Previous results have revealed that cigarette smoke exerts its biological effects via the ERK1/2 signaling pathway (8). Moreover, a critical role for ERK1/2 activation in the abnormal proliferation of vascular smooth muscle cells and airway smooth muscle cells has been suggested during vessel remodeling (11-14). The present study extended this finding and demonstrated that the expression of p-ERK1/2 was upregulated by CSE stimulation, indicating that activated ERK1/2 is involved in the CSE-induced proliferation of primary rPASMCs. When the effect of ERK1/2 on cell cycle distribution was analyzed, consistent with a previous study (29), it was found that activated ERK1/2 promoted cell cycle progression from G0/G1 to $S$ phase. Conversely, ERK1/2-siRNA increased the proportion of cell cycle arrest at the G0/G1 phase.

The molecular mechanism by which ERK1/2 stimulated rPASMC proliferation was also investigated in the present study. Previous studies indicated that nicotine, a critical component in CSE, could upregulate the expression and activity of cyclin E1 in A549 cells and MKN-45 cells $(30,31)$. In the current study, ERK1/2-siRNA decreased the mRNA and protein expression of ERK1/2 and cyclin E1, and inhibited 

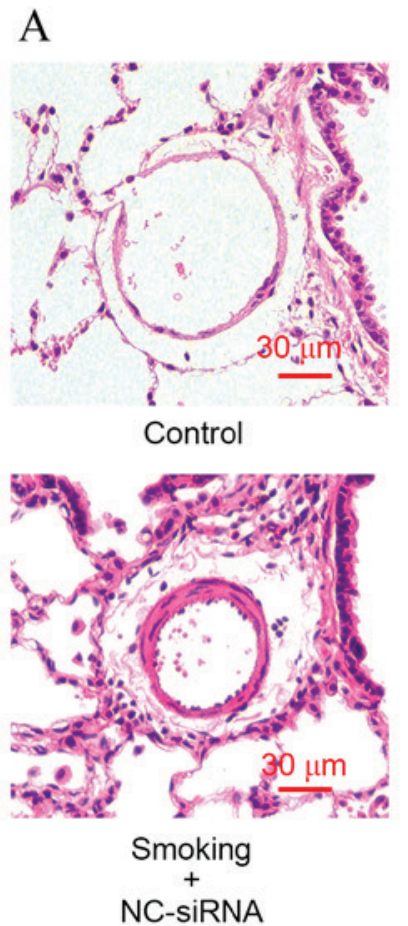

B
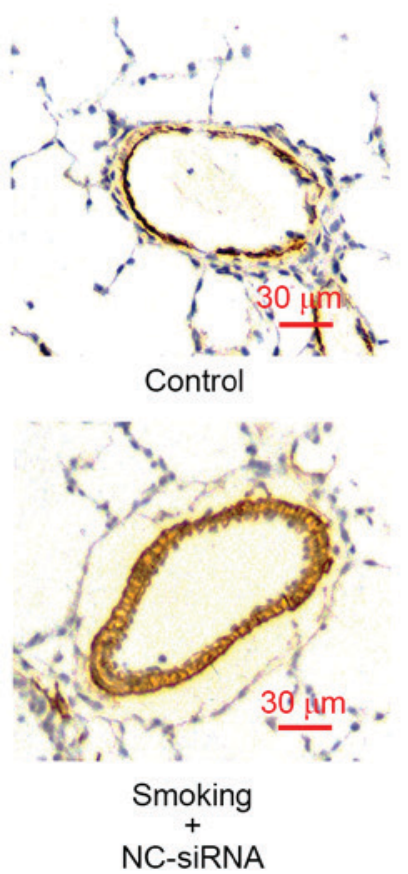
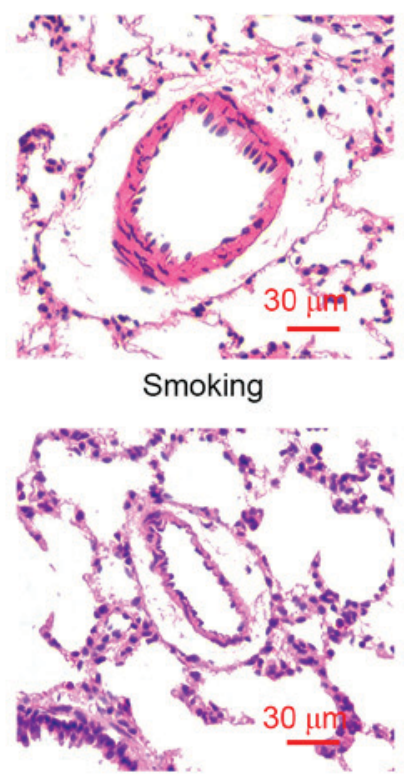

Smoking

ERK1/2siRNA
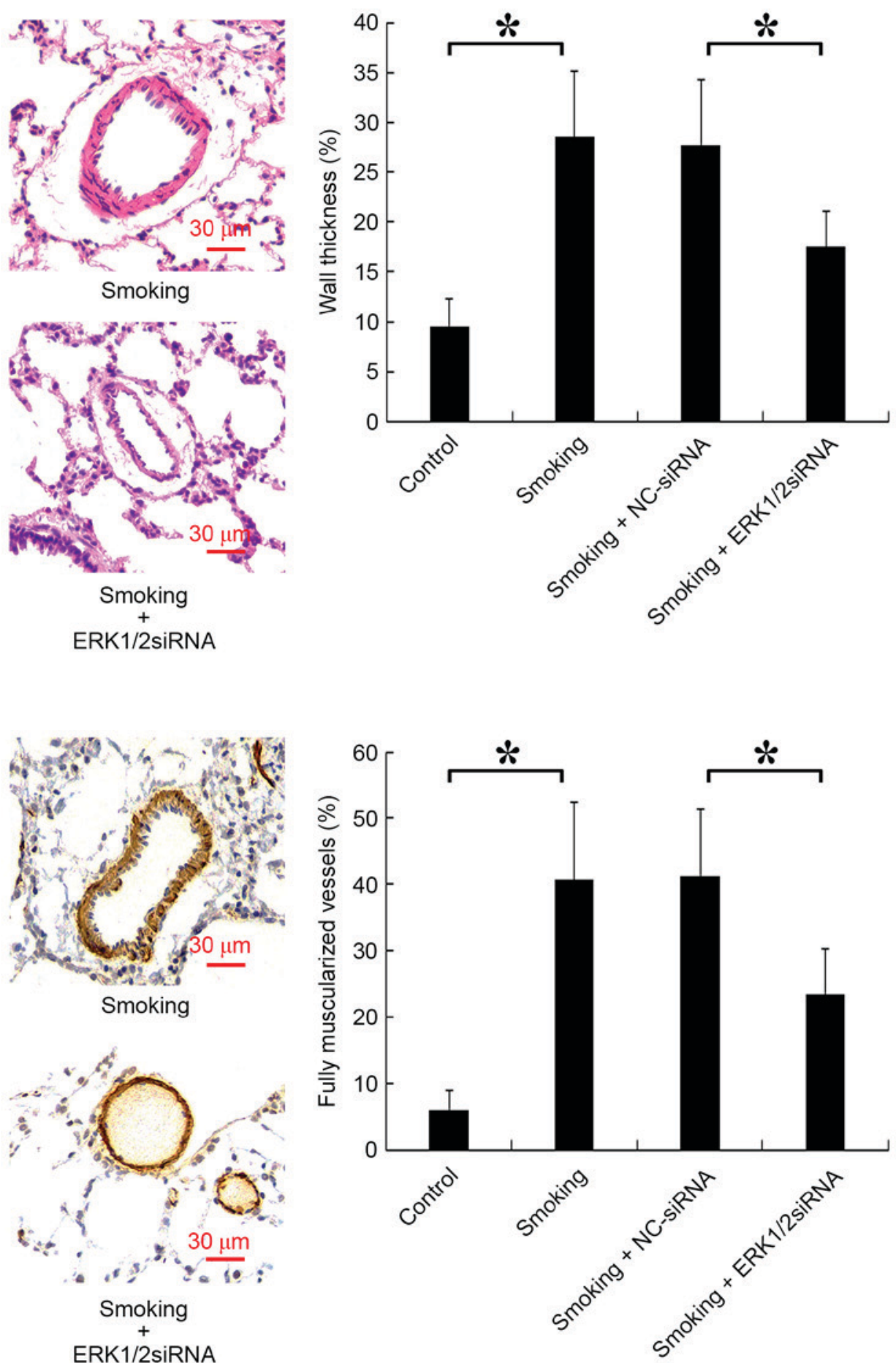

Figure 5. Effect of smoking and ERK1/2-siRNA on rat pulmonary vascular remodeling. Sections were stained with hematoxylin and eosin and anti- $\alpha$-smooth muscle actin antibody. (A) Representative photomicrographs of pulmonary vessel WT are shown (magnification, x200). The WT of different groups were expressed as the percentage of total area in small pulmonary arteries. (B) Representative photomicrographs of the muscularization of small pulmonary arteries are shown (magnification, x200). Blue, indicated the nucleus; red, indicated the cytoplasm; and dark brown, indicated $\alpha$-smooth muscle actin. "P $<0.05$ vs. control. ERK, extracellular signal-regulated kinase; siRNA, small interfering RNA; NC, negative control; WT, wall thickness.

the cell proliferation of CSE-treated rPASMCs. Combined with our previous findings by the current authors that cyclin E1 siRNA significantly decreased rPASMC proliferation (22), these results suggested that CSE could induce rPASMC proliferation by regulating cyclin E1 expression, which was mediated by ERK1/2. These results are also consistent with previous studies in other cell types, which have indicated that ERK1/2 activates cyclin E1 (17-21). In short, ERK1/2 may contribute to rPASMC proliferation, as well as cell cycle progression, and cyclin E1 may be the downstream mediator of ERK1/2 in this process.

PVR is the major feature of pulmonary hypertension in COPD patients (32). In the present in vivo results, increased PVR was observed in cigarette smoke-exposed rats. A 
previous study suggested that ERK1/2 might be involved in this process (33). To elucidate the role of ERK1/2 in PVR in cigarette smoke-exposed rats, ERK1/2-siRNA was delivered by intranasal transfection. As discussed above, the expression of activated ERK1/2 and cyclin E1 were upregulated in the small intrapulmonary arteries of cigarette smoke-exposed rats, and this was effectively attenuated by ERK1/2-siRNA. Based on these results, it was speculated that the mechanism by which ERK1/2 knockdown improves PVR may involve the inhibition of cigarette smoke-induced cyclin E1 expression. Measurements of pulmonary vessel wall thickness and the percentage of fully muscularized arteries revealed that PVR was reduced in cigarette smoke-exposed rats treated with ERK1/2-siRNA. The therapeutic efficacy of ERK1/2-siRNA may be attributed to its inhibitory effect on the proliferation of pulmonary vascular smooth muscle cells of cigarette smoke-exposed rats, as observed in the cell culture study.

It is notable that ERK1/2-siRNA did not completely inhibit the CSE-induced upregulation of cyclin E1 and rPASMC proliferation in vitro, nor did it completely inhibit the enhanced expression of cyclin E1 and PVR in cigarette smoke-exposed rats. These results implied that additional intracellular signaling molecules might be involved in this process, which may include Rb-Raf-1 or protein kinase C $(31,34)$. Indeed, it was also observed that CSE-induced cyclin E1 did not decrease continuously as the ERK1/2-siRNA increase. Therefore, the mechanism by which ERK1/2 regulates cyclin E1 expression requires further clarification.

In conclusion, the present study demonstrated the role of the ERK1/2-cyclin E1 signaling pathway in rPASMC proliferation and PVR of cigarette smoke-exposed rats. The results suggested that cigarette smoke significantly increases rPASMC proliferation and PVR by upregulating the expression of cyclin E1, which is mediated by activated ERK1/2. The inhibition of ERK1/2 with siRNA significantly reduces the expression of ERK1/2 and cyclin E1 both in vitro (CSE-treated rPASMCs) and in vivo (cigarette smoke-exposed rats), and this inhibitory effect contributes to the alleviation of PVR. The results of this study suggest that ERK1/2-siRNA may have therapeutic value in the treatment of COPD.

\section{Acknowledgements}

This study was supported by the National Natural Science Foundation of China (grant nos. 81070044 and 81370156).

\section{References}

1. Churg A, Cosio M and Wright JL: Mechanisms of cigarette smoke-induced COPD: Insights from animal models. Am J Physiol Lung Cell Mol Physiol 294: L612-L631, 2008.

2. Santos S, Peinado VI, Ramírez J, Melgosa T, Roca J, RodriguezRoisin R and Barberà JA: Characterization of pulmonary vascular remodelling in smokers and patients with mild COPD. Eur Respir J 19: 632-638, 2002.

3. Chaouat A, Naeije R and Weitzenblum E: Pulmonary hypertension in COPD. Eur Respir J 32: 1371-1385, 2008.

4. Wright JL, Levy RD and Churg A: Pulmonary hypertension in chronic obstructive pulmonary disease: Current theories of pathogenesis and their implications for treatment. Thorax 60: 605-609, 2005.

5. Peinado VI, Pizarro S and Barbera JA: Pulmonary vascular involvement in COPD. Chest 134: 808-814, 2008.
6. Mandegar M, Fung YC, Huang W, Remillard CV, Rubin LJ and Yuan JX: Cellular and molecular mechanisms of pulmonary vascular remodeling: Role in the development of pulmonary hypertension. Microvasc Res 68: 75-103, 2004.

7. Ferrer E, Peinado VI, Díez M, Carrasco JL, Musri MM, Martínez A, Rodríguez-Roisin R and Barberà JA: Effects of cigarette smoke on endothelial function of pulmonary arteries in the guinea pig. Respir Res 10: 76, 2009.

8. Mercer BA, Kolesnikova N, Sonett J and D'Armiento J: Extracellular regulated kinase/mitogen activated protein kinase is up-regulated in pulmonary emphysema and mediates matrix metalloproteinase-1 induction by cigarette smoke. J Biol Chem 279: 17690-17696, 2004.

9. Mebratu Y and Tesfaigzi Y: How ERK1/2 activation controls cell proliferation and cell death: Is subcellular localization the answer? Cell Cycle 8: 1168-1175, 2009.

10. Ramos JW: The regulation of extracellular signal-regulated kinase (ERK) in mammalian cells. Int J Biochem Cell Biol 40: 2707-2719, 2008.

11. Xie M, Liu XS, Xu YJ, Zhang ZX, Bai J, Ni W and Chen SX: ERK1/2 signaling pathway modulates the airway smooth muscle cell phenotype in the rat model of chronic asthma. Respiration 74: 680-690, 2007.

12. Preston IR, Hill NS, Warburton RR and Fanburg BL: Role of 12-lipoxygenase in hypoxia-induced rat pulmonary artery smooth muscle cell proliferation. Am J Physiol Lung Cell Mol Physiol 290: L367-L374, 2006.

13. Liu B, Ryer EJ, Kundi R, Kamiya K, Itoh H, Faries PL, Sakakibara $\mathrm{K}$ and Kent KC: Protein kinase C-delta regulates migration and proliferation of vascular smooth muscle cells through the extracellular signal-regulated kinase 1/2. J Vasc Surg 45: 160-168, 2007.

14. Ding Q, Gros R, Limbird LE, Chorazyczewski J and Feldman RD: Estradiol-mediated ERK phosphorylation and apoptosis in vascular smooth muscle cells requires GPR 30. Am J Physiol Cell Physiol 297: C1178-C1187, 2009.

15. Morgan DO: Principles of CDK regulation. Nature 374: 131-134, 1995.

16. Möröy T and Geisen C: Cyclin E. Int J Biochem Cell Biol 36: 1424-1439, 2004.

17. Bessard A, Frémin C, Ezan F, Fautrel A, Gailhouste L and Baffet G: RNAi-mediated ERK2 knockdown inhibits growth of tumor cells in vitro and in vivo. Oncogene 27: 5315-5325, 2008.

18. Kisielewska J, Philipova R, Huang JY and Whitaker M: MAP kinase dependent cyclinE/cdk2 activity promotes DNA replication in early sea urchin embryos. Dev Biol 334: 383-394, 2009.

19. Lents NH, Keenan SM, Bellone C and Baldassare JJ: Stimulation of the Raf/MEK/ERK cascade is necessary and sufficient for activation and Thr-160 phosphorylation of a nuclear-targeted CDK2. J Biol Chem 277: 47469-47475, 2002.

20. Keenan SM, Bellone C and Baldassare JJ: Cyclin-dependent kinase 2 nucleocytoplasmic translocation is regulated by extracellular regulated kinase. J Biol Chem 276: 22404-22409, 2001.

21. Lee SH, Park C, Jin CY, Kim GY, Moon SK, Hyun JW, Lee WH, Choi BT, Kwon TK, Yoo YH and Choi YH: Involvement of extracellular signal-related kinase signaling in esculetin induced G1 arrest of human leukemia U937 cells. Biomed Pharmacother 62: 723-729, 2008.

22. Yu MQ, Liu XS, Wu HX, Xiang $M$ and Xu YJ: ERK1/2 promotes cigarette smoke-induced rat pulmonary artery smooth muscle cells proliferation and pulmonary vascular remodeling via up-regulating cyclinel expression. J Huazhong Univ Sci Technolog Med Sci 33: 315-322, 2013

23. Finder JD, Litz JL, Blaskovich MA, McGuire TF, Qian Y, Hamilton AD, Davies P and Sebti SM: Inhibition of protein geranylgeranylation causes a superinduction of nitric-oxide synthase-2 by interleukin-1beta in vascular smooth muscle cells. J Biol Chem 272: 13484-13488, 1997.

24. Livak KJ and Schmittgen TD: Analysis of relative gene expression data using real-time quantitative PCR and the 2(-Delta Delta C(T)) method. Methods 25: 402-408, 2001.

25. Zeng DX, Xu YJ, Liu XS, Wang R and Xiang M: Cigarette smoke extract induced rat pulmonary artery smooth muscle cells proliferation via PKC $\alpha$-mediated cyclin D1 expression. J Cell Biochem 112: 2082-2088, 2011.

26. Carraway MS, Ghio AJ, Suliman HB, Carter JD, Whorton AR and Piantadosi CA: Carbon monoxide promotes hypoxic pulmonary vascular remodeling. Am J Physiol Lung Cell Mol Physiol 282: L693-L702, 2002. 
27. Zhou M, Chen HL, Cheng S, Mei L, Zhang HL, Xie M, Xiong WN and $\mathrm{Xu}$ YJ: Effect of dexamethasone on expression of AGR2 protein in asthmatic mice. J Huazhong Univ Sci Technolog Med Sci 33: 33-36, 2013.

28. Zelko IN, Zhu J, Ritzenthaler JD and Roman J: Pulmonary hypertension and vascular remodeling in mice exposed to crystalline silica. Respir Res 17: 160, 2016.

29. Brondello JM, McKenzie FR, Sun H, Tonks NK and Pouysségur J: Constitutive MAP kinase phosphatase (MKP-1) expression blocks G1 specific gene transcription and S-phase entry in fibroblasts. Oncogene 10: 1895-1904, 1995.

30. Shin VY, Jin HC, Ng EK, Sung JJ, Chu KM and Cho CH: Activation of 5-lipoxygenase is required for nicotine mediated epithelial-mesenchymal transition and tumor cell growth. Cancer Lett 292: 237-245, 2010.

31. Dasgupta P, Rastogi S, Pillai S, Ordonez-Ercan D, Morris M, Haura E and Chellappan S: Nicotine induces cell proliferation by beta-arrestin-mediated activation of Src and Rb-Raf-1 pathways. J Clin Invest 116: 2208-2217, 2006.
32. Sakao S, Voelkel NF and Tatsumi K: The vascular bed in COPD: Pulmonary hypertension and pulmonary vascular alterations. Eur Respir Rev 23: 350-355, 2014.

33. Hu Y, Dietrich H, Metzler B, Wick G and Xu Q: Hyperexpression and activation of extracellular signal-regulated kinases (ERK1/2) in atherosclerotic lesions of cholesterol-fed rabbits. Arterioscler Thromb Vasc Biol 20: 18-26, 2000.

34. Eder AM, Sui X, Rosen DG, Nolden LK, Cheng KW, Lahad JP, Kango-Singh M, Lu KH, Warneke CL, Atkinson EN, et al: Atypical PKCiota contributes to poor prognosis through loss of apical-basal polarity and cyclin E overexpression in ovarian cancer. Proc Natl Acad Sci USA 102: 12519-12524, 2005. 